\title{
Conservación y registro arqueológico en el yacimiento submarino Bucentaure // de La Caleta, Cádiz
}

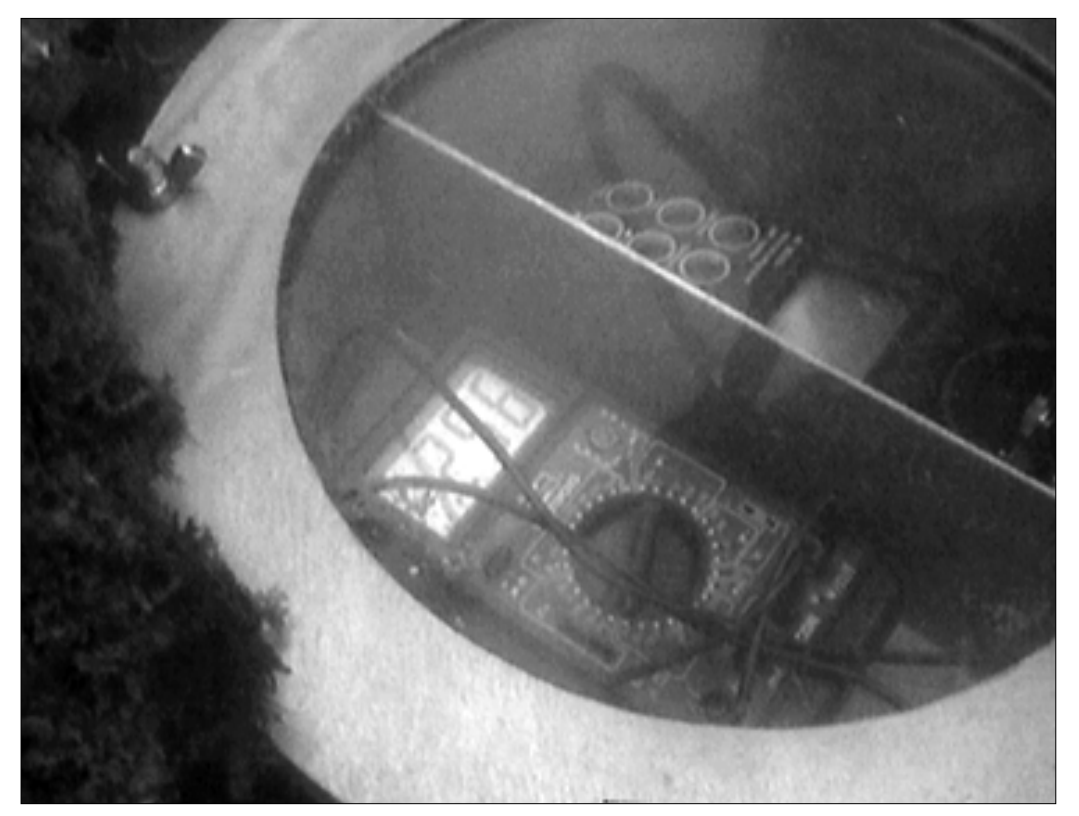

\section{Luis Carlos Zambrano Valdivia} Manuel Bethencourt Núñez

Dpto. de Conservación del Centro de Arqueología Subacuática del IAPH

\section{Resumen}

Una serie de grandes cañones de hierro fuertemente concrecionados en el fondo marino de la Caleta de Cádiz y posiblemente asociados a la Batalla de Trafalgar han servido para la puesta a punto de un sistema combinado de registro arqueológico y control in situ de la corrosión metálica.

Con esta metodología que permite acceder a la información histórica a través de las marcas de fundición existentes en los cañones, sin poner en riesgo su inte- gridad, se pretende aportar un nuevo elemento de utilidad en las técnicas de prospección arqueológicas.

\section{Palabras clave}

Conservación in situ / Corrosión / Concreciones/ Cañones / Registro arqueológico / Artillería naval / Trafalgar; Batalla de

\section{Conservación in situ versus investigación arqueológica}

En muchas ocasiones, la conservación se presenta de forma errónea como un freno a la investigación arqueológica cuyo método es por definición "destructivo" (Coles, J. 1984). Esto ocurre cuando se considera la excavación como la única técnica posible de conoci- 
miento científico para la arqueología. Sin embargo, aunque dicha técnica constituya una herramienta inestimable para "obtener información de evidencia arqueológica" (Price, N.S. 1984). Existen otras técnicas que permiten el conocimiento del Patrimonio Arqueológico Subacuático, como por ejemplo la prospección.

La prospección arqueológica ha experimentado un enorme desarrollo dentro de la moderna arqueología debido tanto a su valor intrínseco como a la política patrimonial (Keller-Rupp, 1983). Este desarrollo se fundamenta sobre dos concepciones de la arqueología aparentemente contrapuestas que son la investigación científica frente a la gestión-protección del Patrimonio Histórico. Afortunadamente, el planteamiento más actual tiende a la formulación de soluciones integradoras (Criado, F. 1996) para superar el absurdo panorama de confrontación entre dos vertientes del mismo objeto.

En la línea de lo expuesto surge este trabajo que pretende aportar un nuevo elemento de utilidad en las técnicas de prospección arqueológica aplicadas tanto a la gestión como al conocimiento científico del Patrimonio.

Mediante la prospección se produce el reconocimiento del sitio arqueológico a través de la fase documental que debe ofrecer un grado de información "suficiente" para interpretar el yacimiento. En este punto es determinante, tanto la calidad como la cantidad de los datos arqueológicos recogidos. Sin embargo, en ocasiones ocurre que los restos materiales localizados en superficie ofrecen una información "insuficiente" para la correcta interpretación del yacimiento.

\section{Sistema combinado de registro arqueológico y control in situ de la corrosión}

Esta circunstancia se produce en aquellos naufragios, donde el único material de superficie se reduce a una serie de grandes cañones recubiertos de concreciones minerales. Si bien es conocida la existencia de marcas y relieves sobre dichos objetos, esta información permanece oculta bajo gruesas capas de concreción que impiden acceder a ella.

Ante ese obstáculo se plantean dos alternativas donde tradicionalmente se contraponen los criterios de conservación e investigación:

a) Renunciar al conocimiento histórico del yacimiento en beneficio de la conservación in situ de los objetos que los componen. En cuyo caso se produce la situación paradójica de conservar y proteger un yacimiento absolutamente desconocido a nivel histórico.

b) Extraer los objetos para su documentación. Esta opción conlleva un alto coste de conservación y la destrucción irreparable del contexto arqueológico difícilmente justificable cuando el interés del objeto se limita a su exclusiva función documental.
Ante esta situación, el Dpto. de Conservación del CAS propone un sistema combinado de desconcreción puntual y control de la corrosión como solución alternativa para obtener una documentación "suficiente" de los objetos conservados en el contexto arqueológico.

El sistema combinado de registro arqueológico y control in situ de la corrosión metálica ha sido puesto en práctica con éxito sobre una serie de grandes cañones de hierro fuertemente concrecionados en el fondo marino de la Caleta de Cádiz. Previamente, la eficacia del sistema ha sido verificada con ensayos a escala de laboratorio sobre material arqueológico desechable.

Dicho sistema aporta ventajas respecto a la calidad del registro obtenido por los métodos tradicionales de documentación subacuática ya que las gruesas capas de concreción mineral distorsionan la forma del objeto metálico impidiendo la observación de marcas y sellos de fundición, así como una medida fiable de sus dimensiones -calibre interior, diámetro y longitud-.

Asimismo, el sistema permite mantener el objeto desconcrecionado en zona de pasivación electroquímica de tal manera que se garantiza su conservación en el contexto arqueológico del yacimiento submarino.

\section{Desconcreción y registro arqueológico}

La desconcreción es una de las intervenciones que componen el repertorio de recursos empleados en la conservación del material arqueológico submarino. Al igual que la excavación arqueológica, se trata de un procedimiento irreversible que debe ser objeto de un registro inteligente (Petrie, 1904) debido a que las diferentes capas de concreción contienen a la vez que ocultan información arqueológica. Para interpretar el objeto se necesita comprender la naturaleza y relaciones existentes entre cada una de sus partes, desde el núcleo metálico hasta la concreción mineral. En este momento metodológico se confunden los objetivos de arqueología y conservación, observándose con claridad la relación simbiótica entre ambas disciplinas (Hamilton, D.L. 1976).

Las gruesas concreciones deformantes son la principal característica de visu que presentan los objetos de hierro fundido, v.g.: los cañones, localizados en yacimientos arqueológicos submarinos. Su formación responde a un proceso originado por la colonización biológica de organismos marinos formadores de material esquelético, principalmente $\mathrm{CaCO}_{3}$, que establece la base inicial de engrosamiento. Esta capa de naturaleza calcárea se alimenta de especies metálicas en difusión $\mathrm{Fe}^{2+}$ y Fe ${ }^{3+}$ procedentes del metal subyacente (North, N.A. 1987) produciendo modificaciones químicas en su composición. Simultáneamente, se experimenta un progreso de la colonización biológica - algas, moluscos y corales -, unida a la precipitación de $\mathrm{CaCO}_{3}$ con inclusiones de sedimento. El resultado 
es una compacta envoltura mineral adaptada a la superficie del objeto. En la práctica, dicha envoltura funciona como un molde rígido, en negativo, que reproduce la forma y el volumen original (fig. I).

En el interior, el objeto sufre un proceso de corrosión electroquímica que transforma el hierro en productos minerales distribuidos en fases uniformes desde el núcleo metálico remanente hasta la zona exterior grafitizada compuesta por $\mathrm{H}_{2} \mathrm{O}, \mathrm{FeO}(\mathrm{OH}), \mathrm{SiO}_{2}, \mathrm{Fe}_{3} \mathrm{C}, \mathrm{Cl}-$ y grafito. Es este último elemento el responsable de mantener la forma primitiva del objeto debido a su particular estructura cristalina de red tridimensional entrelazada (North, N.A. 1987). La zona grafitizada retiene la apariencia del objeto (figura 2 ), -dimensiones, marcas, sellos- pero ha mermado las propiedades físicas del metal-densidad, tenacidad, dureza, etc.-convertido en un agregado de productos minerales.

Así pues, la zona mas interesante a nivel documental es también la que reviste un mayor riesgo de alteración física. Esta circunstancia exige que el trabajo de desconcreción se realice con técnicas mecánicas para ejercer el máximo control sobre la operación. Las herramientas neumáticas ofrecen buenos resultados a nivel de precisión, al tiempo que permiten su empleo bajo agua, conectadas a una botella de aire comprimido. Existe una disponibilidad abundante de máquinas y complementos para efectuar los diversos trabajos que van desde remover en extensión hasta delimitar un área puntual donde se requiera un control minucioso. Las herramientas manuales (cincel, puntero, martillo) permiten un máximo control en la frecuencia e intensidad del impacto percutor, por este motivo se emplean para desprender la concreción previamente delimitada.

El registro arqueológico es el objetivo final del proceso de desconcreción in situ y se produce cuando aparecen datos relevantes de interés arqueológico. Los diferentes métodos de registro están adaptados a la naturaleza del dato arqueológico que pretenda ser documentado, existiendo tantos métodos como tipos de datos.

Los moldes flexibles de silicona se han empleado con éxito en la documentación arqueológica subacuática. Con este método se obtiene un registro tridimensional del objeto que permite un estudio pormenorizado en el laboratorio de arqueología (Zambrano Valdivia, L.C. 2000). Se ha previsto emplear dicho método en el registro de los datos arqueológicos, marcas incisas o sellos en relieve, aparecidos bajo la concreción mineral de los cañones.

\section{Estabilización y control de la corrosión in situ}

La formación de la capa de concreción reduce considerablemente la velocidad de corrosión del hierro en agua de mar, situando al metal en la denominada zona de pasividad del diagrama de Pourbaix. Básicamente, el diagrama de Pourbaix es un método gráfico basado
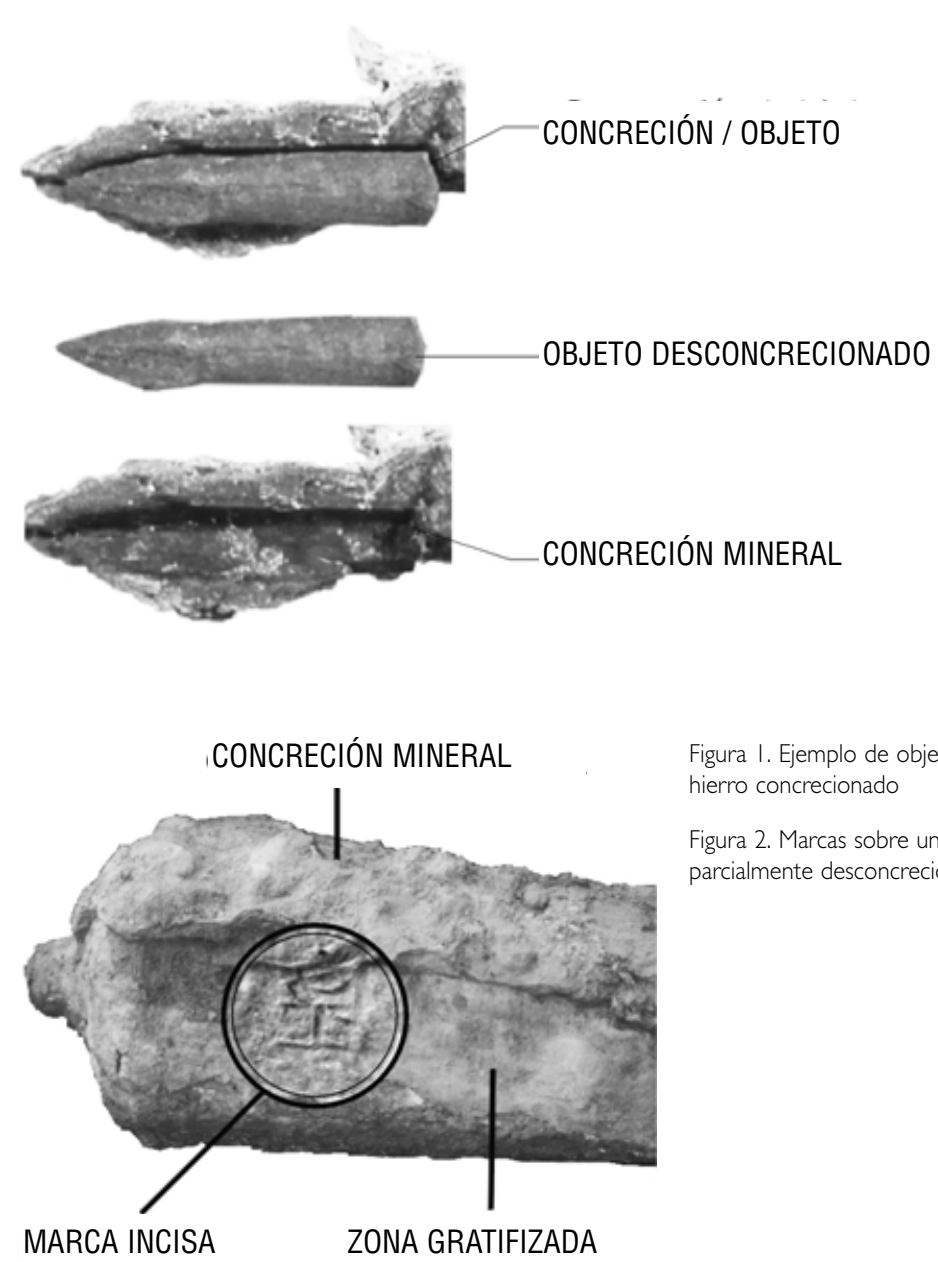

Figura I. Ejemplo de objeto de hierro concrecionado

Figura 2. Marcas sobre un cañón parcialmente desconcrecionado en el empleo de dos parámetros electroquímicos, $\mathrm{pH}$ superficial y potencial de corrosión, $E_{\text {corr. Las medidas }}$ in situ de estos parámetros electroquímicos sobre objetos metálicos presentes en yacimientos arqueológicos subacuáticos han mostrado ser una llave de gran valor para comprender los mecanismos de corrosión de dichos objetos (Gregory, G., 1999).

El diagrama de Pourbaix representa las regiones de estabilidad termodinámica de las especies para los sistemas metal-electrolito (Fontana, M.G., 1978) y permite situar al metal en zona de corrosión, inmunidad o pasividad en un determinado medio. El metal sólo es estable a potenciales inferiores al del equilibrio $\mathrm{Me} \leftrightarrow \mathrm{Me}^{\mathrm{n}}$ + ne-, en la denominada zona de inmunidad de los diagramas. En las restantes zonas la termodinámica predice que el metal tenderá a corroerse pasando al estado combinado, trasformándose en unos u otros productos de corrosión. Únicamente cuando los productos formados son más o menos insolubles, por ejemplo las concreciones, pueden provocar una reducción en la velocidad de corrosión, y en ciertos casos, si se separan totalmente el metal del medio agresivo, garantizar la estabilidad química del mismo.

El desprendimiento de la capa de concreción con fines arqueológicos conllevará la reactivación del proceso corrosivo del metal. Traducido al diagrama de 
Pourbaix, esto supondrá reintroducir el metal en la zona de corrosión, poniendo en peligro la preservación del objeto. Con el fin de proteger al hierro de la corrosión hay que forzarlo a penetrar de nuevo en la zona de pasividad.

Esto se puede conseguir mediante protección catódica con empleo de ánodos de sacrificio. Esta técnica es ampliamente conocida y utilizada para frenar la corrosión de estructuras sumergidas (barcos, pantalanes, tuberías submarinas, etc) o enterradas (tuberías de combustible o contra incendios, depósitos, etc) (González-Fernández, J.A., 1989). Un ánodo de sacrificio consiste, normalmente, en una aleación de cinc o aluminio, conectada eléctricamente al objeto a proteger, compuesto por un metal menos reactivo, como el hierro. El objeto de hierro gana protección como consecuencia de los electrones descargados por la corrosión del ánodo y que fluyen a través del cable de cobre hacia del objeto; es decir, el objeto actúa como cátodo de la celda de corrosión mientras que el agua de mar cierra el circuito. Este efecto disminuye el potencial de corrosión y la velocidad del proceso corrosivo, y permitiría situar al objeto arqueológico desconcrecionado en la zona de inmunidad del diagrama de Pourbaix.

No obstante, el empleo de ánodos de sacrificio presenta una serie de inconvenientes, que en determinadas circunstancias puede desaconsejar su empleo. Por un lado, los costes de operación pueden considerarse medios o altos, requiriendo además un mantenimiento periódico de los ánodos. En aquellos yacimientos donde las visitas se espacien en el tiempo, el empleo de los ánodos está totalmente desaconsejado. Por otro lado, las condiciones de operación deben determinarse empíricamente o basándose en la experiencia previa. La facilidad con la que se puede cometer un error debido a la peculiar naturaleza de los objetos a proteger (peso, volumen y área real desconocidos) puede conducir a procesos de sobreprotección que podrían dar lugar a una excesiva alcalinización en la proximidad del metal con riesgo de deterioro del mismo (RP B40I, 1993).

Otra opción que permitiría volver a situar al hierro en zona de pasividad sería el empleo de un recubrimiento que aislase, al menos en la extensión desconcrecionada, el material metálico del medio agresivo. Este mecanismo, denominado de efecto barrera, se basa en la protección del objeto mediante el aislamiento que se produce al interponer una película sólida y continua entre el metal y el medio corrosivo. Básicamente se persigue impedir que los agentes oxidantes del medio tengan acceso a la superficie metálica, inhibiéndose por lo tanto, la reacción catódica del proceso corrosivo. Sin embargo, la mayoría de los recubrimientos, en mayor o menor extensión, son permeables al agua y al oxígeno, lo cuál debilita la protección conseguida a través de esta vía (Barnhart, R. 1997).

Así, aunque el número de posibles recubrimientos es muy amplio, deben de cumplir con una serie de características: que aseguren la protección mediante una buena permeabilidad, que sean de fácil aplicación y manejo, y que sean económicamente rentables. De entre todas las opciones, los denominados epoxi dos componentes aplicables sobre aceros en la industria (epoxi-poliamidas, epoxi-aminas, epoxis de alquitrán de hulla ó másticos de epoxis, vinílicos, cauchos clorados, y asfálticos) se convierten en la mejor alternativa. Presentan un excelente efecto barrera con una baja o nula porosidad, que impide el intercambio iónico entre el hierro y el electrolito, tienen un buen grado de adherencia a la superficie, y son maleables y fáciles de adaptar sobre cualquier morfología. Presentan además una elevada resistencia a la abrasión y a los agentes agresivos, unido a un bajo coste. En concreto, se ha elegido una resina epoxi de dos componentes empleada en la industria naval para su aplicación en superficies húmedas o sumergidas.

Una vez seleccionado el método de pasivación de las zonas desconcrecionadas, el trabajo se centrará en la medición in situ del pH y del $\mathrm{E}_{\text {corr }}$ para el control de la eficacia del recubrimiento protector a base de resina epoxi.

\section{Material y método}

La desconcreción se ha realizado con herramientas mecánicas y manuales usadas de forma alternativa en función del substrato mineral. La delimitación del área de desconcreción se realiza con el microcincel CTS 178 equipado con grifo de tornillo para regular la intensidad del golpe. Esta herramienta se acciona con aire a presión procedente de una botella de acero de I 5 litros cargada a 250 atmósferas. El suministro se realiza desde la botella a través de una $I^{\text {a }}$ etapa de regulador estándar modificada a 6 atmósferas y una manguera Trelleborg MAXAIR TRI 5045 de 2 metros de longitud. La manguera está roscada a la I ${ }^{a}$ etapa y se une al microcincel mediante una clavija metálica de conexión rápida que permite cambiar fácilmente de herramienta. El efecto de la percusión mecánica sirve para marcar, de forma progresiva, la hendidura que limita la superficie a desconcrecionar. En este trabajo se emplea un protector auditivo estándar de banda ajustable debido a la intensa propagación del sonido en el medio acuático.

En la siguiente fase, se obtiene un control muy preciso mediante cincel y puntero provisto de protector manual, 8747 y 874 I - 250 de ACESA, golpeado con un martillo, 407 "O" de ACESA. Esta herramienta se coloca de forma perpendicular realizando una serie de percusiones firmes para conseguir una fractura limpia en la concreción. Previo a la desconcreción, se efectúa una limpieza de la zona elegida para eliminar algas y depósitos poco adheridos mediante abrasión con cepillo estándar de soldador con el objeto de clarificar el área de trabajo.

El pH se ha determinado usando un electrodo de $\mathrm{pH}$ de superficie plana diseñado para trabajar en tuberías a presiones de hasta 6 bar, modelo HI I00I de Hanna 
Instruments, conectado a un $\mathrm{pH}$-metro de campo modelo HI 9025 de Hanna Instruments. La conexión se efectúa entre ambos mediante el mismo cable del electrodo introducido en un cable flexible goma, que lo aísla del medio. Previamente al desarrollo del trabajo en el yacimiento, el electrodo y el pH-metro son calibrados en el laboratorio del C.A.S. según las normas. El pH-metro incorpora, además, una sonda de temperatura.

La medida del potencial de corrosión $\mathrm{E}_{\text {corr }}$ se realiza empleando electrodos de trabajo y de referencia específicos para la determinación de potenciales en agua de mar. Estos consisten en una sonda de dos componentes: electrodo referencia de $\mathrm{Ag} / \mathrm{AgCl}$ cuyo potencial, respecto al electrodo estándar de nitrógeno es 0.197 voltios, y una varilla de contacto fabricada en acero inoxidable AISI 316. Ambos están conectados a un multímetro digital de alta impedancia modelo M890G de Mastech.

Tanto el multímetro como el pH-metro se sitúan en una carcasa estanca de fabricada en acero inoxidable AISI 316 que permite visualizar los valores de la medida (Figura 3). Esta carcasa ha sido diseñada en el Departamento de Conservación del C.A.S. y permite trabajar a profundidades de hasta 60 metros.

Con el fin de taladrar la concreción depositada sobre los objetos se emplea el taladro neumático modelo 7802 de Ingersoll-Rand, y el martillo neumático modelo $61 \mathrm{H}$ de la misma casa, conectados a una botella de aire comprimido con manómetro regulable, utilizando brocas y puntas de distinto diámetro en función de la necesidad de cada operación (desconcrecionado o conexión del cable de control). En caso de ser necesario, la operación se completa con el uso del cincel manual.

Para posteriores operaciones de control se fija al objeto un cable de control mediante resina epoxi. Este consiste en un tramo de cable de silicona de 5 milímetros de diámetro con cuerpo de cobre de I metros de longitud. El extremo contrario del cable va encapsulado dentro de una rosca de plástico, que evita el contacto del cuerpo de cobre con el agua de mar cuando no se esté utilizando.

Para la cubrición de las zonas desconcrecionadas y la sujeción del cable de control se emplea una resina epoxi de dos componentes miscibles en el agua, Eporaiß | I27/A y Eporaiß I 127/B de IQRaisa Recubrimientos.

\section{Trabajo in situ}

\section{Desconcreción y registro arqueológico.}

Para la desconcreción experimental se escogió el "muñón" de uno de los cañones más accesibles del conjunto. En primer lugar se limpió la zona de algas y concreciones poco adheridas mediante abrasión con el cepillo de soldador para clarificar el área de trabajo. Seguidamente, se marcó una hendidura poco profun-
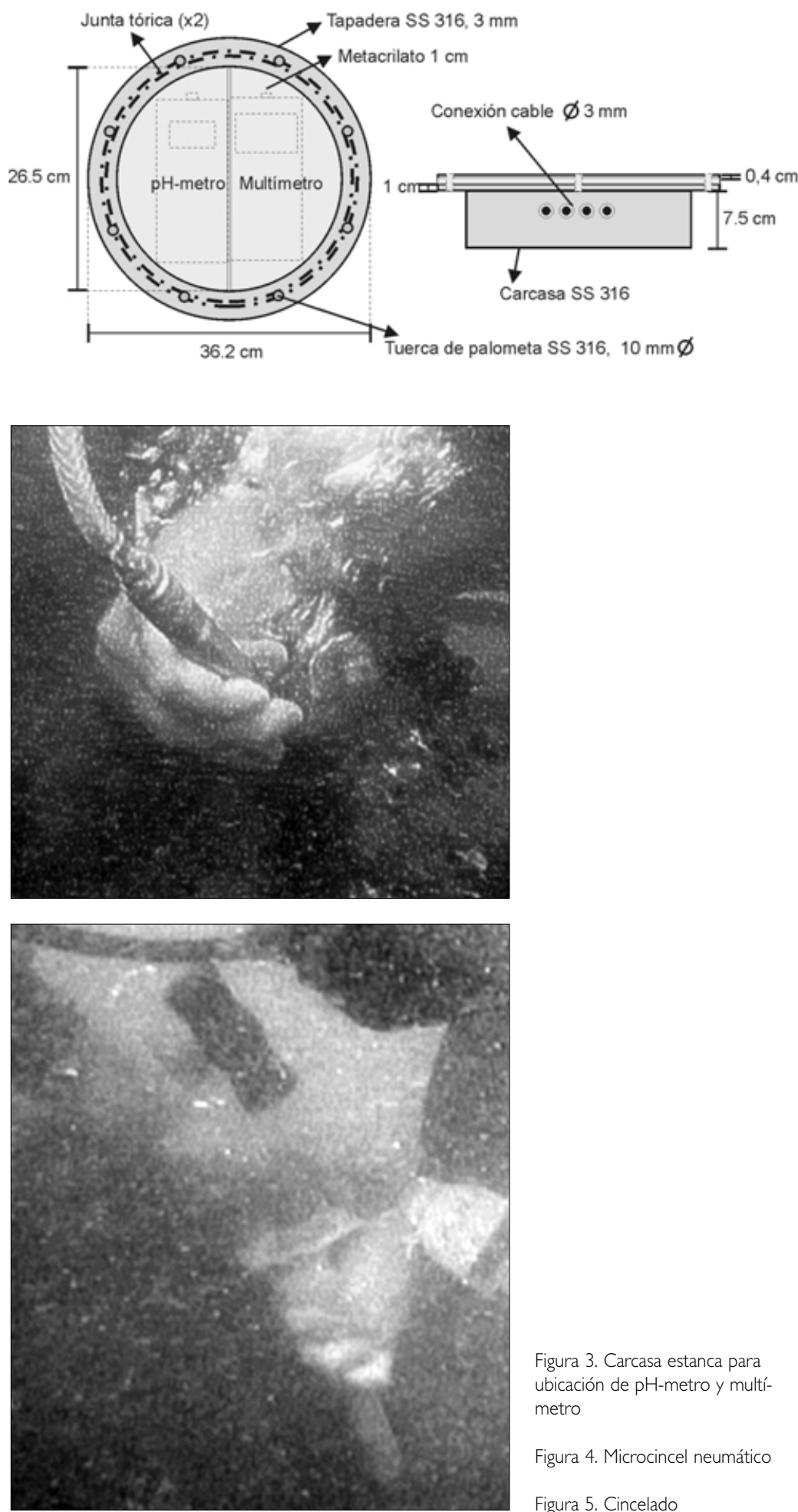

Figura 3. Carcasa estanca para ubicación de $\mathrm{pH}$-metro y multímetro

Figura 4. Microcincel neumático Figura 5. Cincelado da (5- 10 milímetros) con ayuda del microcincel neumático para delimitar la línea de fractura (figura 4).

Una vez completado el perímetro de la zona a remover, se procedió a fracturar la capa de concreción mediante varios golpes de cincel realizados perpendicularmente sobre la anterior hendidura (figura 5).

En este momento se observó la evolución de burbujas desde la interfase concreción / zona grafitizada, 


0
0
1
$-\pi$
5
0
0
0
5
5
0
0
0
0
0
0
4
5
0
1
4

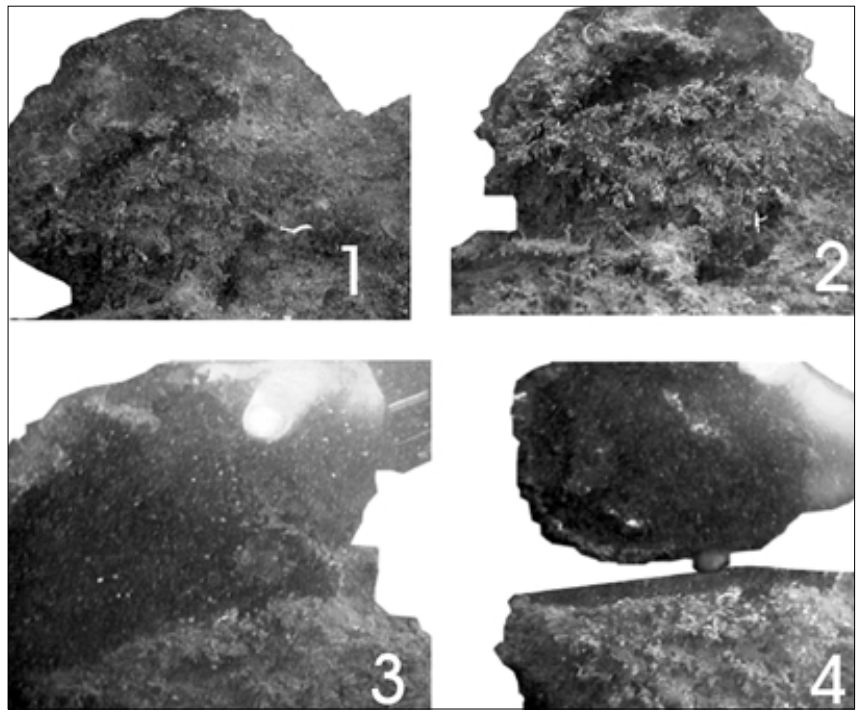

Figura 6. Desconcreción manual del muñón

Figura 7. Medición del pH

Figura 8. Medición de espesores

figura 9. Fijación del cable de control
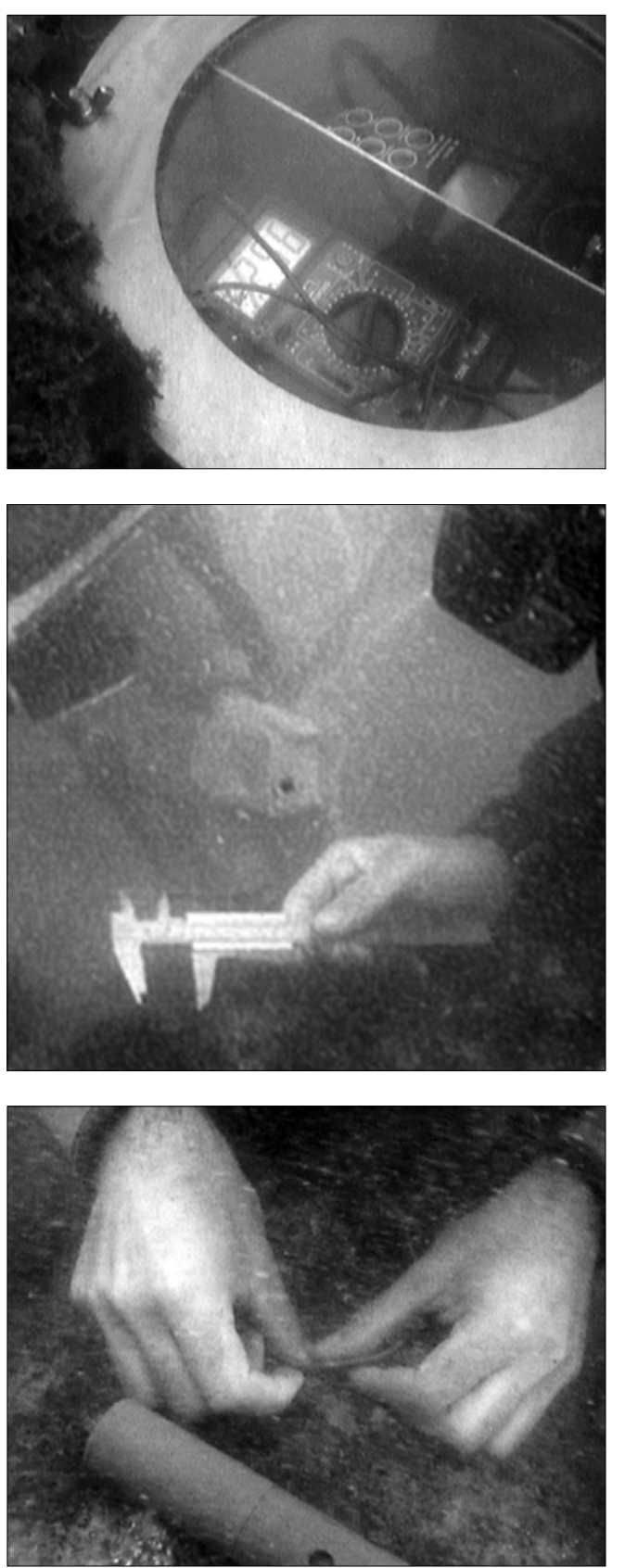

que indicaba la ruptura del sistema estanco de cubrición (Figura I I.I). La concreción se retiro en bloque dejando ver la superficie inalterada de la zona grafitizada que no presentaba ningún tipo de marca o sello de fundición (figura 6).

Por esta razón, quedó inconclusa la fase del registro arqueológico inicialmente prevista mediante moldeo con silicona (figura | I.2).

\section{Toma de datos y conexión del cable de control.}

La actuación para la toma de datos se desarrolló en dos zonas, un muñón y un punto cercano al mismo. Previamente se midió en el entorno del objeto el $\mathrm{pH}_{0}=8.0$ y se recogió una muestra de agua para el posterior análisis de cloruros en el laboratorio, $34.8 \%$.

Se procedió a taladrar la capa de concreción que rodeaba al artefacto hasta alcanzar la superficie metálica (Figura I।.3). El diámetro del taladro no debía superar al diámetro del electrodo de $\mathrm{pH}$.

Una vez realizado el agujero se procedió a limpiar su interior de virutas o restos de concreción con la ayuda de un pincel. El electrodo de $\mathrm{pH}$ se introdujo a través del agujero y se mantuvo próximo a la superficie metálica hasta obtener un valor constante de medida $\mathrm{pH}_{1}=8.0 \mathrm{l}$ (figura 7).

El potencial de corrosión $E_{c o r r, l}$ se midió introduciendo la varilla de acero a través del agujero, de forma que se estableciese contacto eléctrico con la superficie metálica, y situando el electrodo de $\mathrm{Ag} / \mathrm{AgCl}$ en su entorno. El establecimiento de un buen contacto se visualizó mediante la lectura en el multímetro de un potencial estable que fluctuaba de I a $2 \mathrm{mV}$ durante varios minutos y que se situó en $420 \mathrm{mV} \mathrm{Ag} / \mathrm{AgCl}$. Finalmente, el espesor de la concreción se midió introduciendo el extremo reglado de un calibre a través del agujero realizado en la concreción (figura 8). Se obtuvo un espesor de 2.5 cm (Figura |1.4).

Una vez finalizada la toma de datos se procedió a introducir el cable de control y se fijó al objeto mediante resina epoxi de dos componentes (figura 9).

Esta debería evitar el contacto de la superficie metálica con el agua marina y asegurar la sujeción del cable. Se procedió a medir nuevamente el potencial de corrosión, situando el electrodo en las proximidades del agujero sellado y poniendo en contacto la varilla de acero con el cable de control. El potencial $E_{c o r r, 2}$ obtenido fue de $-220 \mathrm{mV} \mathrm{Ag/AgCl}$. Posteriormente se procedió a actuar sobre la zona del muñón. La medida de $\mathrm{pH}_{2}$ se realizó posicionando el electrodo de $\mathrm{pH}$ sobre la zona desconcrecionada hasta obtener un valor constante que coincidía aproximadamente con el medido en la zona taladrada, pHI. La medida del potencial de corrosión se realizó de forma similar a la efectuada anteriormente, y el valor obtenido fue $E_{c o r r, 3}=-306 \mathrm{mV}$. (Figura II.5) 
Una vez realizadas todas las medidas se procedió a cubrir la zona desconcrecionada con resina epoxi (figura 10).

Se midió de nuevo el pH en el entorno del objeto, obteniéndose un valor similar al obtenido inicialmente $\left(\mathrm{pH}_{\circ}\right)$. La medida del potencial de corrosión en el entorno del muñón una vez cubierto con resina, $E_{\text {corr } 4}=$ -240, se realizó poniendo en contacto la varilla de acero con el cable de control, mientras que el electrodo $\mathrm{Ag} / \mathrm{AgCl}$ se situó próximo a la zona cubierta con resina (Figura I I.6).

\section{Interpretación y discusión de los resultados}

Desde el punto de vista arqueológico, la investigación puntual del cañón ha ofrecido un resultado negativo. El plano del muñón desconcrecionado carecía de las presumidas marcas de fundición que hubieran aportado una información relevante para la interpretación del yacimiento. Esta circunstancia ha hecho innecesario el registro arqueológico mediante molde flexible de silicona.

La técnica de desconcreción in situ constituye un éxito metodológico porque ha permitido acceder a la zona grafitizada del cañón sin perjuicio de la misma. Los medios mecánicos se han ejercido con un control adaptado tanto a la naturaleza del material removido como del sustrato remanente. De esta manera se ha logrado una técnica eficaz para romper la compacta concreción mineral en un área determinada, sin alterar el frágil estrato subyacente (zona grafitizada) al que está unido.

Una vez concluida la toma de datos de potencial y $\mathrm{pH}$, se procedió a su interpretación in situ para asegurarnos de la efectividad de la actuación acometida. Mientras, otros datos auxiliares se analizaron en el laboratorio.

En la bibliografía se recogen diferentes diagramas realizados por diversos autores en función de la concentración de cationes metálicos. En nuestro caso hemos utilizado una concentración de 10-6 y hemos adaptado los potenciales al del electrodo de $\mathrm{Ag} / \mathrm{AgCl}$ (figura 12).

El potencial medido a través del agujero realizado sobre la concreción fue $E_{\text {corr, }}=-420$. Situados sobre el diagrama de Pourbaix observamos que este valor se encuentra en la zona de corrosión del hierro. Hay que tener en cuenta que la realización del taladro supone no sólo eliminar la concreción, sino también atravesar la zona grafitizada y llegar al núcleo metálico.

Una vez introducido el cable de control y tras sellar el agujero con la resina, se obtuvo un nuevo valor de potencial, situado en $E_{c o r r, 2}=-220 \mathrm{mV}$. Este valor supone introducir al objeto metálico nuevamente en la zona de pasividad, gracias al empleo de la resina que actúa como película pasivante y protectora.
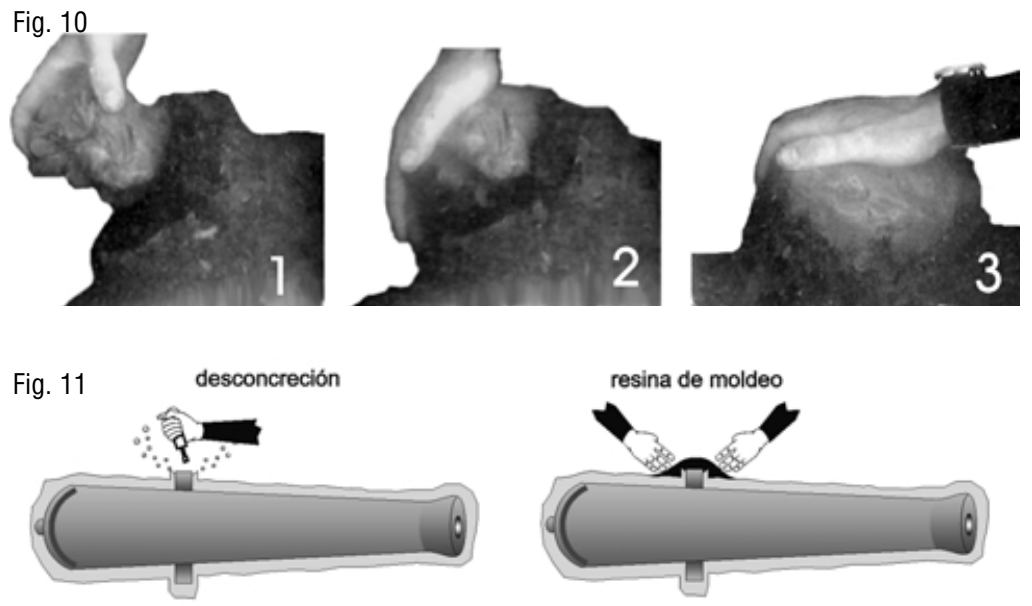

1: Desconcreción del "muñón"

2: Registro mediante molde elástico.

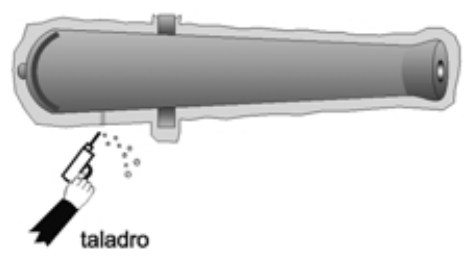

3: Taladro para toma de datos

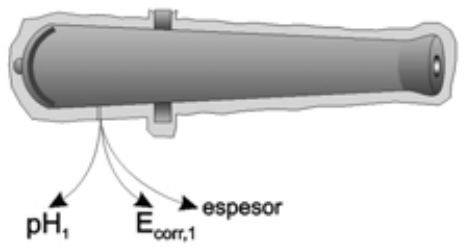

4: Toma de datos iniciales
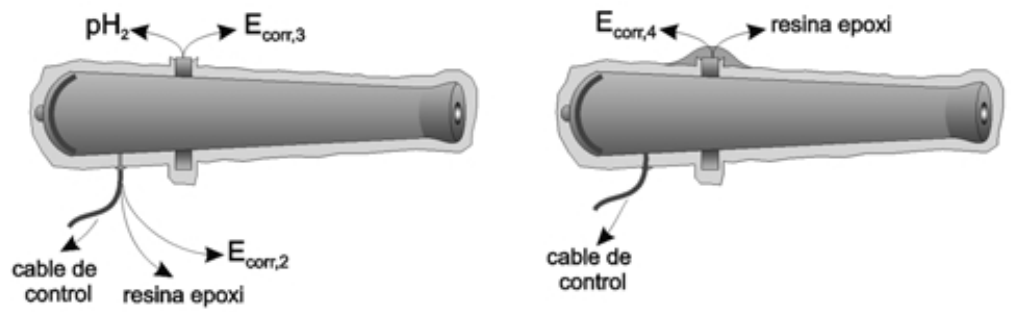

5: Fijación del cable de control y toma de datos

6: Sellado con resina y control de eficacia

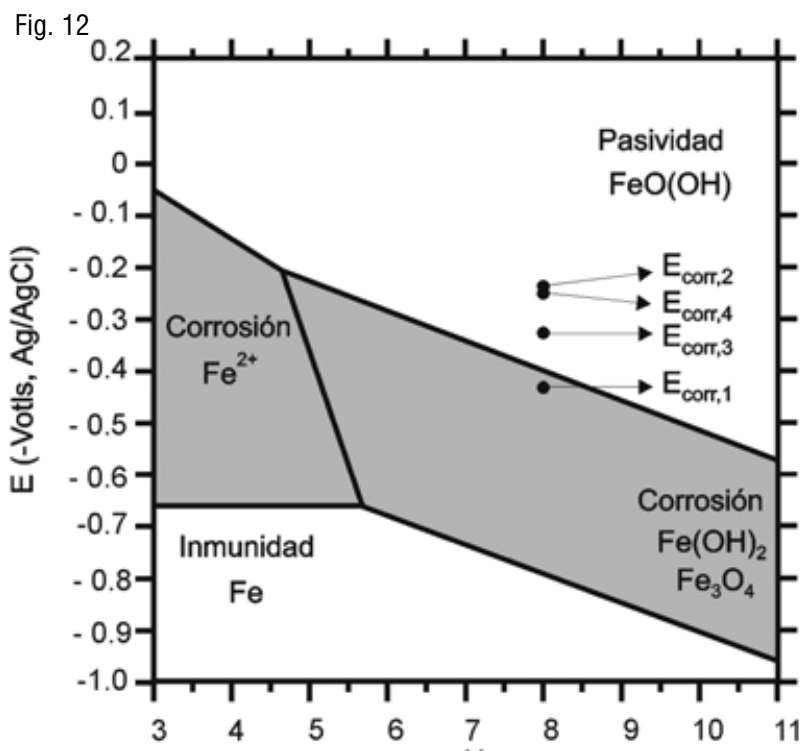

Figura 10. Cubrición del área desconcrecionada con resina epoxi

Figura II. Descripción gráfica del procedimiento

Figura 12. Diagrama de E-pH del hierro en medio acuoso. $25^{\circ} \mathrm{C}$; $10^{-6} \mathrm{M}$. 


\section{Conclusiones}

Se ha diseñado una metodología que permite acceder a la información arqueológica de grandes objetos metálicos concrecionados sin poner en peligro la integridad del mismo. El método es una combinación de dos técnicas, la desconcreción y el control de la corrosión; aplicables a la investigación científica y a la protección-conservación del patrimonio arqueológico submarino.
Se ha realizado una primera experiencia sobre un cañón perteneciente al yacimiento Bucentaure II de La Caleta, Cádiz. El sistema desarrollado permite la eliminación de gruesas capas de concreción que distorsionan u ocultan la información presente en un objeto, proporcionando el acceso a zonas grafitizadas que retienen la apariencia original del mismo. Mediante la desconcreción parcial de uno de los muñones se llegó a dicha zona grafitizada, con resultados negativos en la localización de marcas. No obstante, el acceso a la zona grafitizada sin perjuicio de la misma, se considera como un éxito metodológico.

Posteriormente el trabajo se ha centrado en la medición in situ del pH y del $\mathrm{E}_{\text {corr }}$ para el control del proceso corrosivo que tiene lugar tras la desconcreción. Para ello, los parámetros electroquímicos se han trasladado al diagrama de Pourbaix, que ha servido como herramienta para conocer la evolución del sistema en cada momento del trabajo.

Con el fin de reconducir al objeto a zona de pasividad se ha aplicado una resina epoxi de dos componente que actúa como barrera entre la zona desconcrecionada y el agua de mar, evitando el avance de procesos corrosivos. El control de los parámetros electroquímicos de potencial de corrosión y $\mathrm{pH}$ ha permitido certificar la calidad del recubrimiento utilizado.

\section{Bibliografía}

BARNHART, R. Protective Coatings Europe. 2(8), 1997, pp. | 3- 14.

COLES, J. Documentación y Publicación del Yacimiento. La Conservación en Excavaciones Arqueológicas. ICCROM, Roma, 1984, p. 7I.

CRIADO BOADO, F. La Arqueología del Paisaje como Gestión Integral del Patrimonio Arqueológico. PH: Boletín del IAPH, $\mathrm{n}^{\circ} \mid 4$ 1996, pp. 15-20.

FONTANA, M.G. and GREENE, N.D. Corrosion Engineering En Materials Science and Engineering Series. McGraw-Hill Book Company. 2nd Ed. New York, sec. 9, 1978, pp. 71-79.

GONZÁLEZ-FERNÁNDEZ, J.A. Prevención y protección por técnicas electroquímicas En Control de la Corrosión: Estudio y Medida por Técnicas Electroquímicas, CSIC, Centro Nacional de Investigaciones Metalúrgicas, Madrid, 1989, pp. 236-242.

GREGORY, D. Monitoring the effect of sacrifacialanodes on the large iron artefacts on the Duart Point wreck, 1997. The International Journal of Nautical Archeology, 28:2, 1999, pp. 164-173.

HAMILTON D.L. Conservation of metal objects from underwater sites: a study in methods. Miscellaneus papers, 4, Texas Memorial
Museum. Publication No. I, Texas Antiquities Committee, 1996, pp. 95.

KELLER, D.R. y RUPP, D.W. Archaeological survey in the Mediterranean area, BAR int.series 155, Oxford.

NORTH, N.A. Corrosion products on marine iron. Studies in Conservation, 27, 1982, pp. 75-83.

NORTH, N.A. and MacLeod, I.D. Corrosion of Metals En Conservation of Marine Archaeological Objects, Editor: Colin Pearson, Butterworths Ed., London, 1987, pp. 76-78.

PETRIE, F. Methods and aims in Archaeology. London, 1904, pp.48.

PRICE, N.S. Excavación y Conservación. La Conservación en Excavaciones Arqueológicas. ICCROM, Roma, 1984, p. 13.

RECOMMENDED PRACTICE B40I, Cathodic Protection Desing, Det Norske Veritas Industry AS, Hovik, Norway, 1993, p. 7.

ZAMBRANO VALDIVIA L.C. Moldeo subacuático de objetos arqueológicos. PH: Boletín del IAPH, n³2, 2000, pp. 175-।82. 